\title{
RESOURCE OPTIMIZATION IN THE DECODE-AND-FORWARD HALF-DUPLEX MULTIPLE RELAY-ASSISTED CHANNEL
}

\author{
Adrian Agustin and Josep Vidal \\ Signal Processing and Communications (SPCOM) Group, Technical University of Catalonia (UPC) \\ Email: agustin@gps.tsc.upc.edu, josep.vidal@upc.edu
}

\begin{abstract}
$^{1}$
The half-duplex multiple relay-assisted channel is investigated, where the relays work under decode-and-forward (DF) in time/frequency orthogonal resources. Achievable rates are optimized with respect to the transmitted powers and with respect to the fraction of time/bandwidth allocated to the relay-receive and the relay-transmit phases. Different transmission strategies associated to different complexities at the receivers are considered. For each case we take into account either the synchronous or asynchronous transmission of the terminals, depending on the availability of channel state information (CSI) in transmission from all links. We find that all the cases can be formulated as a unique convex problem, and we compare the achievable rates thus obtained. We finally derive under which conditions the use of two DF relays brings significant gains over the use of one.
\end{abstract}

Index Terms - Relays, cooperative systems, radio spectrum management

\section{INTRODUCTION}

Assisting relays are a promising solution being currently considered to enhance wireless communications in terms of increased data rate and robustness against the channel impairments. It is based on the three-terminal network considered in [1][2], where the relay terminal operates in full-duplex mode. Different coding strategies are analyzed in [3] for several relayassisted/cooperative scenarios where there are multiple sources, relays and/or destinations. In such a case, the relays can work under decode-and-forward or compress-and-forward. However, the full-duplex assumption for the relays is unrealistic in practical systems, and the relay operation must be divided in two orthogonal phases: the relay-receive phase and the relay-transmit phase, according to the mode of the relay. The resources allocated to each of those phases impact on the achievable rate obtained by the relay-assisted transmission. For example, the achievable rates using a single half-duplex relay are investigated in [4][5]. Moreover, in [6] the transmission resources are optimized for maximizing the achievable rate of a multiple access relay-assisted channel with multiple sources, a single destination and half-duplex relays.

In this work we study the achievable rates when two half-duplex relays are present under decode-and-forward, see Fig. 1. The relayassisted transmission is done as follows. The source sends a signal

\footnotetext{
${ }^{1}$ This work was supported by the European Union through projects FIREWORKS IST-4-027675 and ROCKET ICT-2007-1-215282, by the Spanish/Catalan Science and Technology Commissions and FEDER funds from the EC: TEC2006-06481/TCM,TEC2004-04526 and 2005SGR-00639
}

to the relays and destination during the relay-receive phase (solid lines in Fig. 1). In a subsequent phase, and after the relays have decoded and re-encoded the received messages, the source and relays simultaneously transmit during the relay-transmit phase, (dashed lines in Fig. 1). Notice that this protocol is different from the full-duplex transmission defined in [3], where the relays may help between themselves for receiving the messages from the source. The half-duplex constraint at the relays generates a few difficulties when trying to adopt the schemes defined in [3], as how the transmission time between the two phases is selected, how the power is assigned, the optimum messaging or the coordination between relays. One possibility of using two assisting relays is described in [7],[8] where both relays receive and transmit alternatively. On the contrary, here we assume that both relays are receiving or transmitting simultaneously. We study two source transmission strategies: either the relays receive independent messages, or both receive a single common message. In both cases we consider that either the transmitting terminals have a complete CSI of all links (synchronous case ${ }^{2}$ ) so distributed eigenvector precoding techniques can be considered or terminals do not have CSI (asynchronous case).

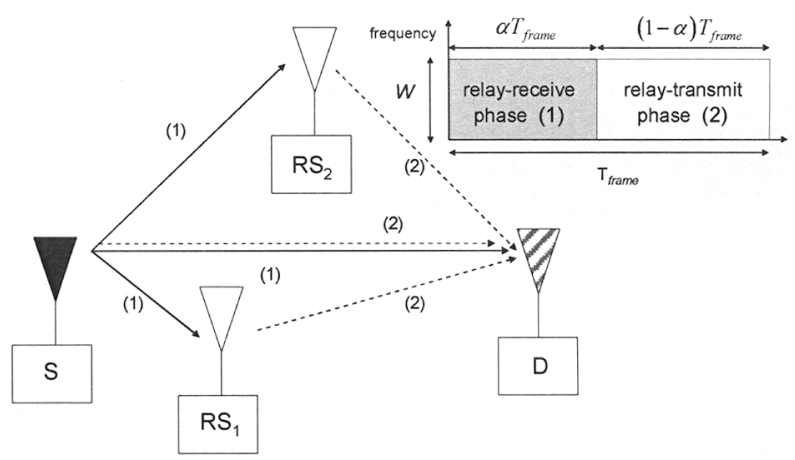

Fig. 1. Multiple half-duplex relay-assisted transmission

The optimum duration of each phase of the transmission and power allocation at the source and relays are obtained by maximizing the achievable rate. We formulate the problem for the case of relays receiving independent messages with CSI. Other cases of practical interest (including the single relay-assisted transmission and the no-CSI case) can be seen as particularizations of that problem.

${ }^{2}$ We adopt the definition from [4]. 


\section{SIGNAL MODEL}

In this work we assume that all terminals are equipped with a single antenna. In the following $\rho_{0}$ denotes the signal to noise (SNR) ratio in the source-destination link, $\rho_{1, \mathrm{i}}$ and $\rho_{2, \mathrm{i}}$ the SNR in the link between the source and the $i$-th relay $\left(\mathrm{RS}_{\mathrm{i}}\right)$ and $i$-th relay and destination, respectively. All these values have been measured using power $P$ at each terminal. The transmission is carried out in frames of length $T_{\text {frame }}(\mathrm{s})$ and bandwidth $B W(\mathrm{~Hz})$ (both parameters are normalized to one). The relays operate in halfduplex mode with a duration $\alpha T_{\text {frame }}$ and $(1-\alpha) T_{\text {frame }}$ for the relayreceive and relay-transmit phase, respectively. We will assume an average sum power constraint for all the terminals. Moreover, if a terminal must keep an average power of $P^{\prime}$ during a period of time of $\alpha T_{\text {frame }}$, it can transmit with power $P^{\prime} /\left(\alpha T_{\text {frame }}\right)$. An extension to the MIMO case should consider the achievable rate of the Broadcast channel (BC) with common information introduced in [9]. This is deferred to a forthcoming work.

The signal received at the relays and destination during the relayreceive phase is $\left(0 \leq t \leq \alpha T_{\text {frame }}\right)$,

$$
\begin{aligned}
& y_{R S_{1}}(t)=\sqrt{\rho_{1,1}} e^{j \theta_{1,1}} x_{0}(t)+n_{R S_{1}}(t) \\
& y_{R S_{2}}(t)=\sqrt{\rho_{1,2}} e^{j \theta_{1,2}} x_{0}(t)+n_{R S_{2}}(t) \\
& y_{D}^{(1)}(t)=\sqrt{\rho_{0}} e^{j \theta_{0}} x_{0}(t)+n_{D}(t)
\end{aligned}
$$

where $n_{D}, n_{R S_{1}}$ and $n_{R S_{2}}$ are the white Gaussian noise of unitary power at the different terminals. The channel gains are expressed in terms of amplitude (square root of the SNR of each link when the noise power is unitary) and the complex phase: $\theta_{0}, \theta_{l, l}$ and $\theta_{l, 2}$ are used for the source-destination, source- $\mathrm{RS}_{1}$ and source- $\mathrm{RS}_{2}$ links, respectively. Finally, $x_{0}$ is the signal transmitted by the source in this phase. Afterwards, the signal received during the relay-transmit phase at the destination is $\left(\alpha T_{\text {frame }}<t \leq T_{\text {frame }}\right)$,

$$
y_{D}^{(2)}(t)=\sqrt{\rho_{0}} e^{j \theta_{0}} x_{3}(t)+\sqrt{\rho_{2,1}} e^{j \theta_{2,1}} x_{1}(t)+\sqrt{\rho_{2,2}} e^{j \theta_{2,2}} x_{2}(t)+n_{D}(t)(2)
$$

where $\theta_{0}, \theta_{2,1}$ and $\theta_{2,2}$ are the complex phases for the sourcedestination, $\mathrm{RS}_{1}$-destination and $\mathrm{RS}_{2}$-destination links, respectively. In this phase the assisting relays transmit the signals $x_{1}$ and $x_{2}$, while the source transmits $x_{3}$. In the following we omit the dependence with the time for the sake of notation simplicity.

\section{INDEPENDENT MESSAGES}

In the scenario depicted in Fig. 1 we assume that the relay-assisted transmission is done by conveying independent messages to the relays. The transmission consists of three messages $W_{1}, W_{2}$ and $W_{3}$, at rates $R_{1}, R_{2}$ and $R_{3}$, respectively. The first two messages use the help of the relays, while the third message is sent only by the source during the relay-transmit phase. The messages have associated different Gaussian codebooks. Table 1 depicts how the messages, terminals and codewords are connected along with the fractions of total average power $P$ used by each terminal. There, $\gamma_{0, \mathrm{i}}$ with $i=\{1,2,3\}$ stands for the fraction of total average power used by the source for transmitting the codewords $m_{1}^{(1)}, m_{2}^{(1)}, m_{3}^{(2)}$. The assisting relays which have decoded the messages $W_{l}$ and $W_{2}$, only transmit $m_{1}^{(2)}, m_{2}^{(2)}$ along with the source. The variable $\varphi_{j, i}$ with $j=\{0,1,2\}$ and $i=\{1,2\}$ defines the fraction of average power allocated by the $j$-th terminal ( $j=0$ corresponds to the source) to the $i$-th codeword. This is a general description, where some of the variables could be set directly to zero depending on the scenario definition, i.e. if $\mathrm{RS}_{1}$ is able to decode both messages and $\mathrm{RS}_{2}$ just the second one, then $\varphi_{2,1}$ will be zero.

\begin{tabular}{|c|c|c|c|c|c|c|}
\hline Messages & \multicolumn{2}{|c|}{$\boldsymbol{W}_{1}$} & \multicolumn{2}{c|}{$\boldsymbol{W}_{\mathbf{2}}$} & \multicolumn{2}{c|}{$\boldsymbol{W}_{\mathbf{3}}$} \\
\hline Phase & $\boldsymbol{R R}$ & $\boldsymbol{R T}$ & $\boldsymbol{R R}$ & $\boldsymbol{R T}$ & $\boldsymbol{R R}$ & $\boldsymbol{R T}$ \\
\hline codewords & $m_{1}^{(1)}$ & $m_{1}^{(2)}$ & $m_{2}^{(1)}$ & $m_{2}^{(2)}$ & $\times$ & $\boldsymbol{m}_{3}^{(2)}$ \\
\hline \hline$S$ & $\gamma_{0,1}$ & $\varphi_{0,1}$ & $\gamma_{0,2}$ & $\varphi_{0,2}$ & $\times$ & $\gamma_{0,3}$ \\
\hline$R S_{1}$ & $\times$ & $\varphi_{1,1}$ & $\times$ & $\varphi_{1,2}$ & $\times$ & $\times$ \\
\hline$R S_{2}$ & $\times$ & $\varphi_{2,1}$ & $\times$ & $\varphi_{2,2}$ & $\times$ & $\times$ \\
\hline
\end{tabular}

Table 1. Fraction of average power $P$ allocated by each terminal to each codeword used in each phase (RR for relay-receive and RT for relay-transmit phase). The power used by $m_{1}^{(1)}$ is $\gamma_{0,1} P /\left(\alpha T_{\text {frame }}\right)$.

\subsection{Messages and codewords}

During the relay-receive phase only the source is active, broadcasting messages $W_{1}$ and $W_{2}$ to the relays $\mathrm{RS}_{1}$ and $\mathrm{RS}_{2}$ using Gaussian signals of average power $\gamma_{0,1} P$ and $\gamma_{0,2} P$ respectively, see Table 1. Therefore the signal transmitted by the source $x_{0}$ in this period of time, see (1), can be written as,

$$
x_{0}=\sqrt{\gamma_{0,1}} m_{1}^{(1)}+\sqrt{\gamma_{0,2}} m_{2}^{(1)}
$$

with $m_{1}^{(1)}, m_{2}^{(1)}$ being the Gaussian codewords of average power $P$ associated to messages $W_{1}$ and $W_{2}$,

In our single antenna scenario, we may assume that the relayreceive phase is a degraded channel [11], so one of the relays will be able to decode both messages, while the other relay will only decode one. During the relay-transmit phase the former relay can transmit signals connected to both messages. For example, if $\mathrm{RS}_{1}$ decodes both messages, then it will transmit two Gaussian signals with average power $\varphi_{1,1} P$ and $\varphi_{1,2} P$ conveying messages $W_{1}$ and $W_{2}$ respectively, while $\mathrm{RS}_{2}$ will transmit a signal of average power $\varphi_{2,2} P$ connected to the message $W_{2}$ and $\varphi_{2,1}$ in Table 1 will be zero. In the limiting case where the channel state from the source to both relays is equal, only one message is transmitted.

During the relay-transmit phase the source will transmit two Gaussian signals with average power $\varphi_{0,1} P$ and $\varphi_{0,2} P$ devoted to help the transmission from the relays (see Table 1), as well as a new Gaussian signal of average power $\gamma_{0,3} P$ connected to an independent message $W_{3}$. The Gaussian signals transmitted from the different terminals in (2) are,

$$
\begin{aligned}
& x_{1}=\sqrt{\varphi_{1,1}} m_{1}^{(2)}+\sqrt{\varphi_{1,2}} m_{2}^{(2)} \\
& x_{2}=\sqrt{\varphi_{2,1}} m_{1}^{(2)}+\sqrt{\varphi_{2,2}} m_{2}^{(2)} \\
& x_{3}=\sqrt{\varphi_{0,1}} m_{1}^{(2)}+\sqrt{\varphi_{0,2}} m_{2}^{(2)}+\sqrt{\gamma_{0,3}} m_{3}^{(2)}
\end{aligned}
$$

where $m_{1}^{(2)}, m_{2}^{(2)}, m_{3}^{(2)}$ are the Gaussian codewords of average power $P$ associated to $W_{1}, W_{2}$ and $W_{3}$. It is worth noticing that equation (4) describes the general case. However, for the degraded 
channel where $\mathrm{RS}_{1}$ is better than $\mathrm{RS}_{2}$, then $\varphi_{2,1}=0$ because it cannot decode the message $W_{1}$. If $\mathrm{RS}_{2}$ is better than $\mathrm{RS}_{1}$, then $\varphi_{1,2}=0$ because it cannot decode the message $W_{2}$.

In order to obtain the spectral efficiency per unit total power, the average power allocated to the different Gaussian codewords in (3) and (4) must satisfy the average sum power constraint

$$
\sum_{j=1}^{3} \gamma_{0, j}+\sum_{j=1}^{2}\left(\varphi_{0, j}+\varphi_{1, j}+\varphi_{2, j}\right)=1
$$

in which the instantaneous transmitted power at the source (relay) terminals is inversely proportional to the fraction of time allocated to the first (second) phases. In this regard, the different transmission schemes use the same total average power. Other power constraints can be considered, for example an individual power constraint at the source and a sum-power constraint for all the relays. Its evaluation is tackled in a forthcoming work.

\subsection{Achievable rate region}

The achievable rate region described here depends on the maximum decodable rates of messages $W_{1}$ and $W_{2}$ at the relays during the relay-receive phase (denoted by the rate region $\mathcal{R}_{\mathrm{RS}}$ ), as well as the decodable rates of messages $W_{1}, W_{2}$ and $W_{3}$ at the destination during both phases of the transmission (denoted by the rate region $\mathcal{R}_{\mathrm{D}}$ ). In the first case, the rates of the messages $W_{1}$ and $W_{2}$ are defined by the degraded broadcast channel (BC) [11] to $\mathrm{RS}_{1}$ and $\mathrm{RS}_{2}$ transmitting the Gaussian codewords $m_{1}^{(1)}$ and $m_{2}^{(1)}$. Assuming that $\rho_{1,1} \geq \rho_{1,2}$ the data rates are given by

$$
\mathcal{R}_{R S}=\left\{\begin{array}{l}
R_{1} \leq \alpha \log _{2}\left(1+\frac{\rho_{1,1} \gamma_{0,1}}{\alpha}\right) \\
R_{2} \leq \alpha \log _{2}\left(1+\frac{\rho_{1,2} \gamma_{0,2}}{\alpha\left(1+\rho_{1,2} \gamma_{0,1}\right)}\right)
\end{array}\right.
$$

where $\alpha$ is the duration of the relay-receive phase. Notice that in this case, $\mathrm{RS}_{1}$ is able to decode both messages while $\mathrm{RS}_{2}$ only can decode message $W_{2}$. The decodable rates of the different messages (using the codewords $m_{1}^{(2)}, m_{2}^{(2)}$ and $m_{3}^{(2)}$ ) can be seen as a Multiple Access Channel (MAC) with three terminals [11], but taking also into consideration the signal received during the relayreceive phase at the destination. The achievable rate region is given by,

$$
\mathcal{R}_{\mathcal{D}}=\left\{\begin{array}{l}
R_{1} \leq f\left(\gamma_{0,1} \rho_{0}\right)+g\left(\kappa_{1}\right) \\
R_{2} \leq f\left(\gamma_{0,2} \rho_{0}\right)+g\left(\kappa_{2}\right) \\
R_{3} \leq g\left(\gamma_{0,3} \rho_{0}\right) \\
R_{1}+R_{2} \leq f\left(\left(\gamma_{0,1}+\gamma_{0,2}\right) \rho_{0}\right)+g\left(\kappa_{1}+\kappa_{2}\right) \\
R_{1}+R_{3} \leq f\left(\gamma_{0,1} \rho_{0}\right)+g\left(\kappa_{1}+\gamma_{0,3} \rho_{0}\right) \\
R_{2}+R_{3} \leq f\left(\gamma_{0,2} \rho_{0}\right)+g\left(\kappa_{2}+\gamma_{0,3} \rho_{0}\right) \\
\sum_{j=1}^{3} R_{j} \leq f\left(\left(\gamma_{0,1}+\gamma_{0,2}\right) \rho_{0}\right)+g\left(\kappa_{1}+\kappa_{2}+\gamma_{0,3} \rho_{0}\right)
\end{array}\right.
$$

with the following definitions

$$
\begin{gathered}
f(x)=\alpha \log _{2}\left(1+\frac{x}{\alpha}\right) \quad g(x)=(1-\alpha) \log _{2}\left(1+\frac{x}{1-\alpha}\right) \\
\kappa_{1}=\left(\sqrt{\varphi_{1,1} \rho_{2,1}}+\sqrt{\varphi_{0,1} \rho_{0}}\right)^{2} \\
\kappa_{2}=\left(\sqrt{\varphi_{2,2} \rho_{2,2}}+\sqrt{\varphi_{1,2} \rho_{2,1}}+\sqrt{\varphi_{0,2} \rho_{0}}\right)^{2}
\end{gathered}
$$

where $\kappa_{1}$ and $\kappa_{2}$ are the average signal power of the Gaussian codewords $m_{1}^{(2)}$ and $m_{2}^{(2)}$ obtained from the assumption of synchronous transmission of the terminals (which amounts to distributed eigenvector precoding). The terms $1 / \alpha$ and $1 /(1-\alpha)$ in (8) stand for the increment of instantaneous power due to the fraction of time allocated to each phase, keeping the average power constraint. Here, it has assumed that $\varphi_{2,1}=0$ because $\mathrm{RS}_{2}$ is not able to decode message $W_{1}$. Otherwise, the roles of $\mathrm{RS}_{1}$ and $\mathrm{RS}_{2}$ are exchanged.

\subsection{Optimizing the resource allocation}

The destination must decode the messages $W_{1}, W_{2}$ and $W_{3}$ transmitted by the source and relays, so the resource allocation (which involves the duration of each phase $\alpha$, and the power of the different codewords at the different terminals) can be obtained as the result of the following optimization problem,

$$
\begin{aligned}
& \max _{\mathbf{z}} R_{1}+R_{2}+R_{3} \quad \text { s.t. } \quad\left(R_{1}, R_{2}, R_{3}\right) \in B_{\text {ind }}(\mathbf{z})=\left\{\mathcal{R}_{R S} \cap \mathcal{R}_{D}\right\} \\
& \mathbf{z}=\left[\alpha, \gamma_{0,1}, \gamma_{0,2}, \gamma_{0,3}, \varphi_{0,1}, \varphi_{0,2}, \varphi_{1,1}, \varphi_{1,2}, \varphi_{2,1}, \varphi_{2,2}\right] \\
& \alpha \in[0,1] \sum_{j=1}^{3} \gamma_{0, j}+\sum_{j=1}^{2}\left(\varphi_{0, j}+\varphi_{1, j}+\varphi_{2, j}\right)=1
\end{aligned}
$$

where $B_{\text {ind }}$ is the rate region defined by the inequalities (6) and (7). This problem is not convex [10] due to the inequalities of $\mathcal{R}_{\mathrm{RS}}$ in (6) which correspond to the achievable rate region of a degraded broadcast channel (in that case the capacity region). However, in our scenario, we can use the duality BC-MAC [12] to define the achievable rate region at the relays as,

$$
\mathcal{R}_{R S}=\left\{\begin{array}{r}
R_{1}+R_{2} \leq \alpha \log _{2}\left(1+\frac{\lambda_{1} \rho_{1,1}}{\alpha}+\frac{\lambda_{2} \rho_{1,2}}{\alpha}\right) \\
\text { s.t. } \lambda_{1}+\lambda_{2}=\gamma_{0,1}+\gamma_{0,2}
\end{array}\right.
$$

where the optimization is done over the variables $\lambda_{1}$ and $\lambda_{2}$. Those variables are related to $\gamma_{0,1}$ and $\gamma_{0,2}$ in the following way,

$$
\gamma_{0,1}=\lambda_{1} \frac{1}{1+\rho_{1,2} \lambda_{2}} \quad \gamma_{0,2}=\lambda_{2}\left(1+\frac{\rho_{1,2} \lambda_{1}}{1+\rho_{1,2} \lambda_{2}}\right)
$$

Finally, the optimization problem given by $(10)$ with $B_{\text {ind }}$ defined by (11), (12) and (7) becomes convex.

\section{OTHER TRANSMISSION STRATEGIES}

The transmission scheme presented above requires the use of successive cancellation receivers at the relay and destination terminals, as well as the knowledge of the channel state for all links, which may lead to some practical implementation problems in terms of complexity and information feedback. In the following some simplified strategies are detailed, which can be seen as particular cases of the transmission scheme defined in section 3 . 


\subsection{Asynchronous transmission}

When there is no CSI the terminals should transmit asynchronously. In such a case, the transmission cannot benefit of the distributed eigenvector precoding. The source does not help in the transmission of the messages sent by the relays in the relaytransmit phase and the destination cannot combine coherently the signals sent by the different terminals connected with the same codeword. This implies the following changes in (10),

$$
\varphi_{0,1}=\varphi_{0,2}=0, \quad \kappa_{2}=\varphi_{2,2} \rho_{2,2}+\varphi_{1,2} \rho_{1,2}
$$

\subsection{Relays receiving a common message}

The use of simple receivers imply forcing all the assisting relays to receive (and hence transmit) a common message, hence the transmission is constrained by the worst source-relay link (S-RS in our definition). The achievable rates at the relays are given by

$$
\mathcal{R}_{R S}=\left\{\begin{array}{l}
R_{2} \leq f\left(\rho_{1,1} \gamma_{0,2}\right) \\
R_{2} \leq f\left(\rho_{1,2} \gamma_{0,2}\right)
\end{array}\right.
$$

Additionally, the modifications to be considered for the synchronous and asynchronous transmission are,

$$
\begin{aligned}
& \text { Sync }\left\{\begin{array}{l}
\gamma_{0,1}=0, \quad \kappa_{1}=0, \quad R_{1}=0, \quad \varphi_{0,1}=\varphi_{2,1}=\varphi_{1,1}=0 \\
\kappa_{2}=\left(\sqrt{\varphi_{0,2} \rho_{0}}+\sqrt{\varphi_{1,2} \rho_{2,1}}+\sqrt{\varphi_{2,2} \rho_{2,2}}\right)^{2}
\end{array}\right. \\
& \text { Async }\left\{\begin{array}{l}
\gamma_{0,1}=0, \quad \kappa_{1}=0, \quad R_{1}=0, \\
\varphi_{0,1}=\varphi_{0,2}=\varphi_{2,1}=\varphi_{1,1}=0, \kappa_{2}=\varphi_{1,2} \rho_{2,1}+\varphi_{2,2} \rho_{2,2}
\end{array}\right.
\end{aligned}
$$

\subsection{Single relay}

The achievable rates for the single relay case are easily found by considering one assisting relay (e.g. $\mathrm{RS}_{1}$ ). The particularizations for synchronous and asynchronous cases are,

$$
\begin{gathered}
\text { Sync }\left\{\begin{array}{l}
\gamma_{0,2}=0, \quad \kappa_{2}=0, \quad R_{2}=0, \quad \varphi_{0,2}=\varphi_{2,1}=\varphi_{2,2}=0 \\
\varphi_{1,2}=0, \quad \kappa_{1}=\left(\sqrt{\varphi_{0,1} \rho_{0}}+\sqrt{\varphi_{1,1} \rho_{2,1}}\right)^{2}
\end{array}\right. \\
\text { Async }\left\{\begin{array}{l}
\gamma_{0,2}=0, \quad \kappa_{2}=0, \quad R_{2}=0, \quad \varphi_{1,2}=0 \\
\varphi_{0,1}=\varphi_{0,2}=\varphi_{2,1}=\varphi_{2,2}=0, \quad \kappa_{1}=\varphi_{1,1} \rho_{2,1}
\end{array}\right.
\end{gathered}
$$

\section{RESULTS}

The relay-assisted protocols defined previously are evaluated in a Gaussian channel scenario where the source-destination distance is normalized to $1 . \mathrm{RS}_{1}$ and $\mathrm{RS}_{2}$ are placed at distance $d_{1}$ and $d_{2}$ from the source, and $\left(1-d_{1}\right)$ and $\left(1-d_{2}\right)$ from the destination. The pathloss of each link is proportional to the square of the distance between the terminals. The SNR of the different links is given by defining $\rho_{0}$ (source-destination link),

$$
\rho_{1, i}=\frac{P}{\sigma^{2}} \frac{1}{d_{i}^{2}} \quad \rho_{2, i}=\frac{P}{\sigma^{2}} \frac{1}{\left(1-d_{i}\right)^{2}} \quad \rho_{0}=\frac{P}{\sigma^{2}} \quad i=\{1,2\}
$$

The signal model defined in (1) and (2) will be assumed with the previous SNR definition. In our scenario the complex phases of the channels are equal to zero, $\left(\theta_{0}, \theta_{1,1}, \theta_{1,2}, \theta_{2,1}\right.$, and $\left.\theta_{2,2}\right)$ but this value only is known at the transmitters under CSI.

Fig. 2 presents the achievable rate for the transmission using a single relay placed at different positions when CSI is available (square-line) or not (circle-line). Those rates are compared with synchronous and asynchronous transmission of independent or common messaging with two relays, being $\mathrm{RS}_{2}$ placed at $d_{2}=0.2$. The best performance is obtained by the synchronous transmission of independent messages. When both relays are at the same distance $d_{1}=d_{2}=0.2$, independent and common messaging coincide. However, in certain configurations the adoption of a common messaging strategy provides no rate gains compared to the use of a single relay case, even when CSI is considered. The main drawback of common messaging is that the achievable rate is dominated by the relay with the worst channel quality. For the CSI case, there is a rate gain over a single relay case for $0 \leq d_{l} \leq 0.65$. In that region, the relay-destination link limits the communication but thanks to the synchronous transmission of the two relays the equivalent link is improved. The source-relay channel limits the performance when $\mathrm{RS}_{1}$ is close to the source and then $\mathrm{RS}_{2}$ is not helpful. For the asynchronous transmission, common messaging does not offer any rate gain ${ }^{3}$ for $0.2 \leq d_{l} \leq 1$. However, for those positions of $\mathrm{RS}_{1}$, independent messaging under asynchronous transmission improves the single relay case because the position of $\mathrm{RS}_{1}$ does not limit the total transmission.

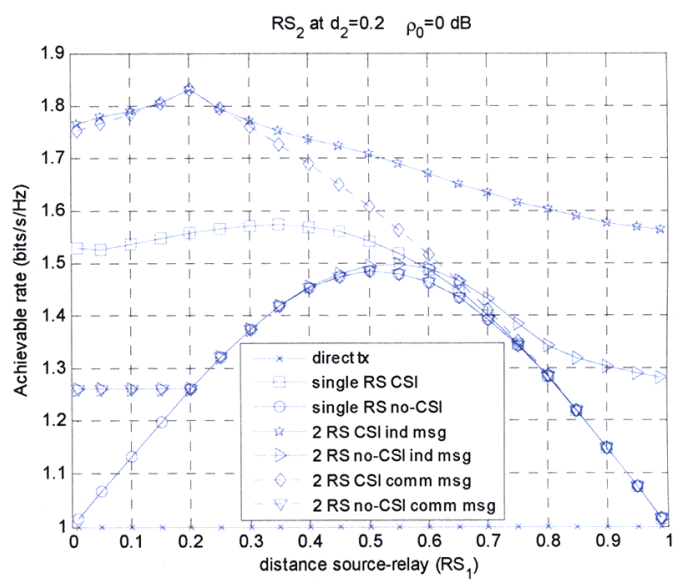

Fig. 2. Achievable rate for the single and 2-relays with/without CSI $v s$ distance between source- $\mathrm{RS}_{1}, \mathrm{RS}_{2}$ at $d_{2}=0.2 . \rho_{0}=0 \mathrm{~dB}$

In order to analyze in more detail what happen with the independent messaging, Fig. 3 sketches the rate of the different codewords as a function of the position of $\mathrm{RS}_{1}$, being $\mathrm{RS}_{2}$ placed at $d_{2}=0.2$. The codeword rate of message $W_{3}$ is zero for both cases for this scenario and it has not been included in Fig. 3. $R_{1}$ and $R_{2}$ stand for the codeword rate through $\mathrm{RS}_{1}$ and $R S_{2}$, respectively. When $0 \leq d_{1} \leq 0.2, \mathrm{RS}_{1}$ is able to decode the message intended to $\mathrm{RS}_{2}$. Under the CSI case (synchronous), $R_{2}$ increases with $d_{1}$,

\footnotetext{
${ }^{3}$ When the channel phases are random (as in fading channels), outage capacity gains are observed.
} 
while $R_{1}$ converges to zero at $d_{l}=0.2$. In such a case, a single message is transmitted to the relay with the worst channel link quality. Hence the performance of common and independent messaging is the same (see Fig. 2). A similar conclusion can be obtained for no-CSI case (asynchronous), where $\mathrm{R}_{1}$ is set to 0 . When $0.2<d_{1} \leq 1$ the role of $\mathrm{RS}_{2}$ and $\mathrm{RS}_{1}$ are exchanged, that explains the discontinuity of the codeword rates at $d_{l}=0.2$. The codeword rates are adapted as a function of $d_{l}$.

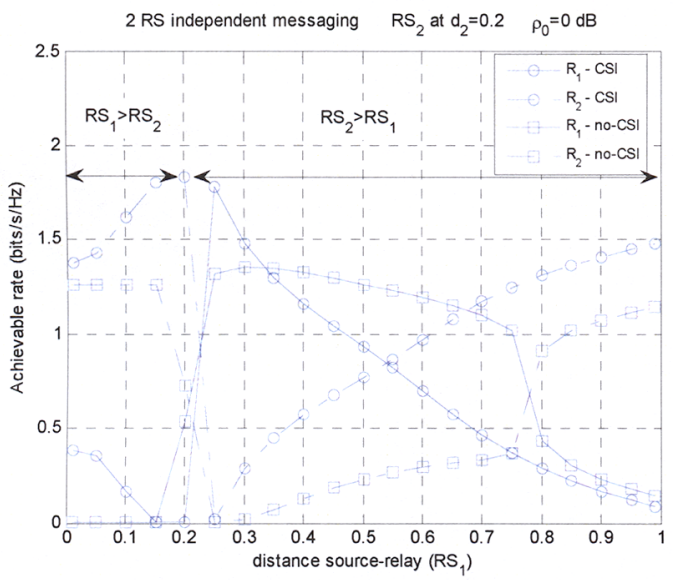

Fig. 3. Achievable rate of the messages for 2-relays with/without CSI $v s$ distance between source- $\mathrm{RS}_{1}, \mathrm{RS}_{2}$ at $d_{2}=0.2 \cdot \rho_{0}=0 \mathrm{~dB} \cdot R_{3}=0$.

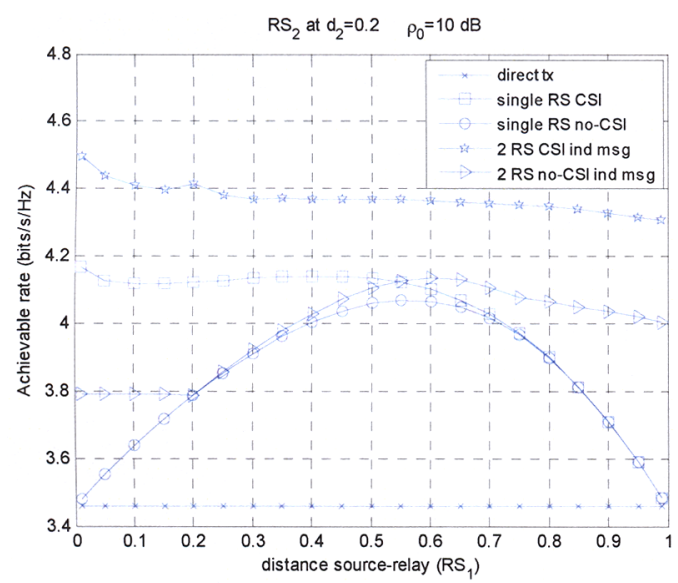

Fig. 4. Achievable rate for the single and 2-relays with/without CSI $v s$ distance between source- $\mathrm{RS}_{1}, \mathrm{RS}_{2}$ at $d_{2}=0.2$. $\rho_{0}=10 \mathrm{~dB}$

Fig. 4 present results of independent messaging with $\rho_{0}=10 \mathrm{~dB}$ and the $\mathrm{RS}_{2}$ placed at $d_{2}=0.2$. This technique with CSI exhibits a performance which is almost independent of the position of $\mathrm{RS}_{1}$, and superior to the single relay case. Additionally, even if CSI is not considered, the use of an additional relay outperforms single relay transmission when $R S_{1}$ is close to the destination. In such a case, the achievable rate for the single relay case is limited by the source-relay link. However, the use of two relays (one near of the source and other close to destination) together with the optimization of allocated power and duration of the relaytransmission phases may deal with that drawback.

\section{CONCLUSIONS}

We have explored the achievable rate gains of half-duplex multiple relay transmission schemes based on decode-and-forward, under different conditions of CSI and decoding complexity. The resource allocation problem based on rate optimization is shown to be convex. It is found that for the Gaussian channel assumed in this work, the use of multiple relays outperforms the single relay case when synchronous transmissions are possible, due to the eigenvector precoding. The optimal power used by the terminals and the duration of the phases of the relay-assisted transmission are adapted to the topology of the network. When one of the relays is placed near the source, independent messaging with synchronous transmission allows a quasi constant achievable rate irrespective of the position of the other relay.

\section{REFERENCES}

[1] E.C. van der Meulen, "Three-terminal communication channels", Adv. Appl. Prob., vol. 3, pp. 120-154, 1971.

[2] T.M.Cover, A.A. El Gamal, "Capacity theorems for the relay channel", IEEE Trans. on Information Theory, vol. 25, no. 5 pp. 474-584, Sept. 1979.

[3] G.Kramer, M.Gastpar, P.Gupta, "Cooperative strategies and capacity theorems for relay networks", IEEE Trans. on Information Theory, vol. 51, no. 9, Sept. 2005.

[4] A.Høst-Madsen, J.Zhang, "Capacity bounds and power allocation for wireless relay channels", IEEE Trans. on Information Theory, vol. 51, no. 6, June 2005.

[5] Y. Liang, V. Veeravalli, "Gaussian Orthogonal Relay Channels: Optimal Resource Allocation and Capacity", IEEE Trans. on Information Theory, vol. 51, no. 9, Sept 2005.

[6] A.Agustin, J.Vidal, "Radio Resources optimization for the Half-duplex Relay-assisted Multiple Access Gaussian Channel", in Proc. $8^{\text {th }}$ IEEE Workshop on SPAWC, Helsinki, Finland, June 2007

[7] B.Rankov, A.Wittneben, "Spectral efficient protocols for half-duplex fading relay channels", IEEE Journal on Sel. Areas in Communications, vol.25, no.2, Feb. 2007

[8] Y.Fan, C.Wang, J.Thompson, H.Vincent Poor, "Recovering multiplexing loss through successive relaying using repetition coding", IEEE Trans. on Wireless Communications, vol. 6, no. 12, Dec. 2007

[9] H. Weingarten, Y. Steinberg, S. Shamai, "On the Capacity Region of the Multi-Antenna Broadcast Channel with Common Messages", in Proc. IEEE ISIT 2006, Seattle, USA, July 2006

[10] S.Boyd, L.Vandenberghe, Convex optimization, Cambridge University Press, 2004.

[11] T.Cover, J.Thomas, Information Theory. New York, Wiley, 1991.

[12] S.Vishwanath, N.Jindal, A.Goldsmith, "On the duality of Gaussian Multiple-Access and Broadcast channels", IEEE Trans. on Information Theory, vol. 50, no. 5, Oct. 2004. 